\title{
Triage of patient phone calls in inflammatory bowel disease clinic: decreasing the administrative burden and improving the patient experience
}

\begin{abstract}
Aim: To assess the volume of calls, identify the major types of inquiries and implement changes in delivery of care.

Methods: All patient phone calls to the inflammatory bowel disease (IBD) clinic were collected retrospectively for a period of one month (March 2016). These were categorized by types of concern. Based on the analysis of these calls, workflow changes such as informational "biologics packets", encouragement of use of electronic portal, subspecialization of the workflow of the nurse coordinators and partnership with specialty pharmacies/infusion center were implemented. Patient calls were collected again for one month (May 2017), to assess impact of the above interventions.
\end{abstract}

Results: A total of 418 phone calls and 185 phone calls were recorded during February 2016 and May 2017, respectively. Majority of the callers were female, with $61 \%$ of calls in February 2016 and 55\% calls in May 2017. Most common diagnosis among the patients was Crohn's disease; $48 \%$ and $43 \%$ of the patient callers had this diagnosis in Feb 2016 and May 2017 respectively. A decrease in the number of calls in each grouping category (including those associated with high administrative burden such as inquiries related to scheduling, insurance, medication orders, lab orders and medical records) was noted in May 2017 as compared to February 2016. A significant number of calls (37\% in March 2016 and $26 \%$ in May 2017) did not need direct input from clinical providers.

Conclusion: Evaluation of the patients' calls to characterize the common reasons and implementing workflow changes based on the analysis can help decrease administrative burden.

Keywords: inflammatory bowel disease clinic, quality improvement; phone call triage, specialty pharmacy, infusion center
Volume II Issue I - 2020

\author{
Anand Nath, Tenzin Choden, Aimee Le \\ Strange, Mark C Mattar \\ Department of Gastroenterology, Medstar Georgetown \\ University Hospital, USA
}

\section{Correspondence: Anand Nath, Department of Gastroenterology, Medstar Georgetown University Hospital, USA, Tel +I-202 6894836, Email anandnath I@gmail.com}

Received: December 24, 2019 | Published: January 31, 2020
Abbreviations: IBD, inflammatory bowel disease; ED, emergency department; EMR, electronic medical record; PDSA, plan-do-study-act

\section{Introduction}

Inflammatory bowel disease (IBD) is a chronic disease of the gastrointestinal system that affects more than 1.6 million patients in the United States. ${ }^{1}$ It is associated with a significant financial burden on the healthcare system. ${ }^{2}$ Additionally, as compared to other gastrointestinal diseases, IBD patients have higher medical care utilization. ${ }^{3,4}$ Management of complex chronic diseases such as IBD requires regular office visits, occasional emergency department (ED) visits and hospitalizations, as well as frequent inter-visit communications between patients and healthcare providers, pharmacies, and insurers. Given the complexity of diagnosis and treatment involved in IBD, both patients and providers, including nurses and support staff, face many challenges including patient literacy in understanding their disease and adherence to treatment.

Most tertiary IBD centers have a call center-based communication to optimize the care of IBD patients. Results have shown that such contact-center based communication is helpful in continued care of these patient. ${ }^{5}$ However, patient phone calls in a tertiary IBD clinic contribute significantly to the workflow of daily clinical practice.
Information on volume of calls, time spent on telephone activity and reasons for calls in the care of IBD patients is sparse currently. ${ }^{6,7}$ Follow up of patients' active medical problems, new onset symptoms, refill requests, insurance authorization for specialty medication, form completion and medical record requests are some of the common reasons for telephone calls in a U.S. tertiary IBD centers. ${ }^{7}$

At our center (a tertiary IBD center at a university hospital in an urban setting), providers observe a large volume of daily patient inquiries, which result in an estimated average of 5hours of follow up to address. The effort devoted to adequately address these issues leads to a significant investment of time on the part of the provider and support staff, as well as re-routing of clinical workflow. The aim of this study is to identify and categorize the major types of inquiries made by patients, to implement changes in delivery of care and streamline provider workflow based on the analysis of the major types of inquiries, and to assess impact of these changes on the number and type of patient phone calls.

\section{Materials and methods}

Approval for human studies was obtained from the GeorgetownMedStar Institutional Review Board. This is a retrospective, single institution study performed in a large, urban community hospital. 
The study was conducted in two separate phases, including a preliminary background data collection phase and then a plan-dostudy-act (PDSA) cycle. In the background data collection phase, all patient calls answered by the IBD nurse coordinator were recorded by providers via the electronic medical record (EMR) for one month, during February of 2016 (29 days). These were subsequently categorized by types of concern for grouping purposes. The categories include:

a. Disease related updates such as improvement or worsening of existing symptoms, onset of new symptoms or requirement of urgent medical attention etc.

b. Calls with clinical questions such as questions related to medication dose, side effects and potential interactions, procedure related questions, questions related to plan of care etc.

c. Request of call back from clinical providers

d. Inquiries related to scheduling of appointment, imaging, next infusion of biologics etc.

e. Inquiries related to medication refills or orders such as request for refills, change in pharmacy etc.

f. Inquiries related to lab results, update of results availability or request for lab orders etc.

g. Inquiries related to insurance such as billing issues, medication coverage, prior authorizations etc.

h. Calls related to medical records such as release of medical records or update of medical records

i. Calls with complaints

j. Others such as update related to non-disease related procedure, request for referral, request for letter etc.

Some calls were related to multiple issues and were counted into each of the corresponding categories. The calls were grouped into various categories independently by two authors. Any disagreement was resolved by discussion. Physician and nurse providers were polled on the duration of time expended on each type of concern, in order to estimate the weight of each of these categories. On review of the most common reasons for phone calls, both the most time-consuming category (prior authorizations for medications) and the most frequent reason for phone calls (scheduling) were chosen for intervention.

The main problem identified with the influx of infusion-related calls was that our center did not have an internal infusion center, and patients were choosing various local infusions centers. Patients had to call the center in order to set up an infusion and to facilitate the medication order to be sent to the specific infusion center. In order to target this issue, the following interventions were implemented:

i. Patients who started new biologic therapy were given a "biologics packet" which contains an IBD welcome letter, information on infusion and injection instructions, and signatures needed for financial assistance programs.

ii. A direct partnership was established with one specific outpatient infusion center to facilitate the scheduling and transmission of infusion orders.

iii. Prior authorization calls had the highest time burden cost according to the providers. Given the number of new medications for IBD requiring prior authorizations, our goal was to streamline the process to decrease the burden on providers. Following intervention was implemented to address this:

iv. The IBD clinic started working with specialty pharmacies to help decrease the burden of prior authorization for injectable biologics.

v. Additional interventions to improve the patients' understanding of their disease and medications, and to decrease the number of calls related to non-urgent issues that occurred during our PDSA cycle included the following:

vi. Patients were given information about electronic patient portal and encouraged to communicate through it for non-urgent issues.

vii. New nurse coordinators were hired, which lead to subspecialization of the workflow of the nurse coordinators.

viii. "Biologics packet" also included information related to the medication side effects. Patient calls taken by the IBD nurse coordinators were recorded again for a one-month period (May 2017; 31 days), in order to assess any impact of our interventions on distribution and volume of the calls to the IBD clinic. These calls were again grouped into categories using the previously mentioned methodology.

De-identified patient information was obtained on a Microsoft Excel sheet. The Microsoft Excel sheet was password protected and stored on a password protected computer. Statistical analysis and computing was performed using Microsoft Excel. Demographics table as well as comparison graphs were generated using Microsoft Excel.

\section{Results}

A total of 418 phone calls and 185 phone calls were recorded during February 2016 and May 2017, respectively. Majority of the callers were female, with $61 \%$ of calls in February 2016 and 55\% calls in May 2017 (Table 1). Most common diagnosis among the patients was Crohn's disease; $48 \%$ and $43 \%$ of the patient callers had this diagnosis in Feb 2016 and May 2017 respectively (Table 1). We have identified disease related updates, clinical questions, scheduling and medication orders/refills to be the most common reasons for patients' telephone calls. Decrease in the number of calls in each grouping category was noted in May 2017 as compared to February 2016 (Figure 1). A large number of calls, such as inquiries related to scheduling, inquiries related to insurance, requests for medical records and complaints (37\% in March 2016 and 26\% in May 2017) did not need direct input from clinical providers. Percentage distribution of the calls related to various categories in the respective months is shown in Figure 2.

Table I Patient demographics

\begin{tabular}{lll}
\hline \multicolumn{2}{l}{ Patient demographics } \\
\hline Sex & Feb 20I6 $(\mathrm{n}=418)$ & May 20I7 $(\mathrm{n}=185)$ \\
\hline Male & $163(39 \%)$ & $84(45 \%)$ \\
Female & $255(61 \%)$ & $101(55 \%)$ \\
\hline IBD patients & \\
\hline Crohn's disease & $202(48 \%)$ & $80(43 \%)$ \\
Ulcerative colitis & $105(25 \%)$ & $63(34 \%)$ \\
Others & $110(26 \%)$ & $42(23 \%)$ \\
\hline
\end{tabular}




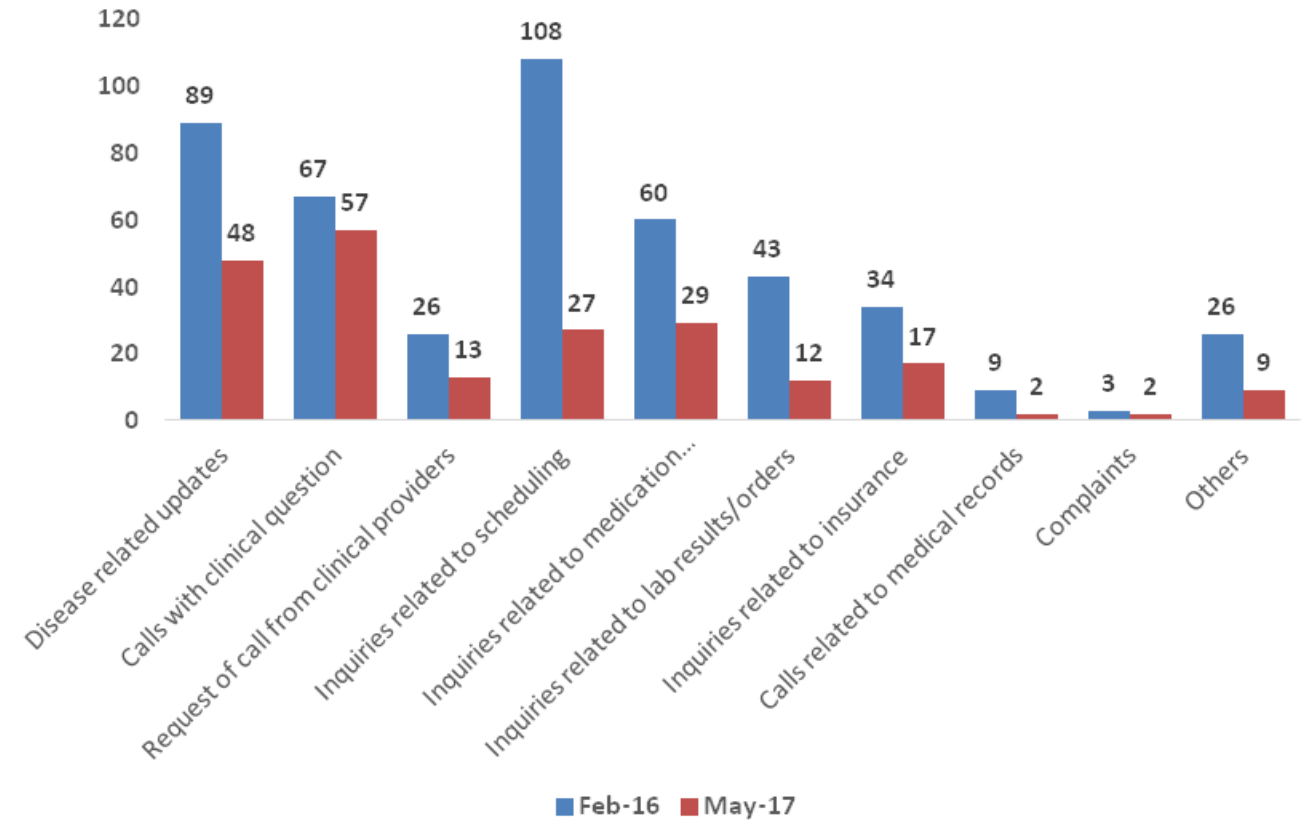

Figure I Absolute number of phone calls related to the different categories in February 2016 and May 2017.

Feb 2016

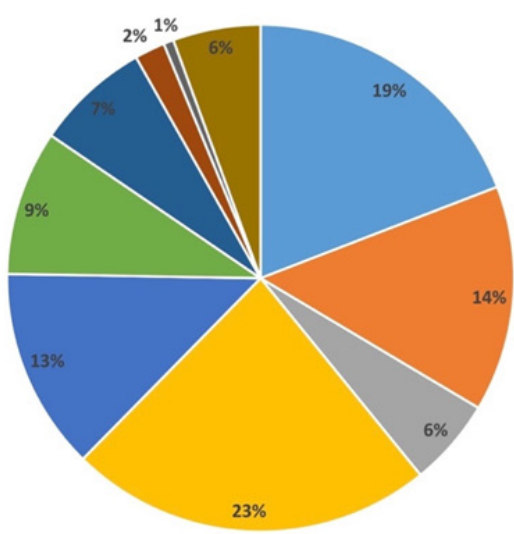

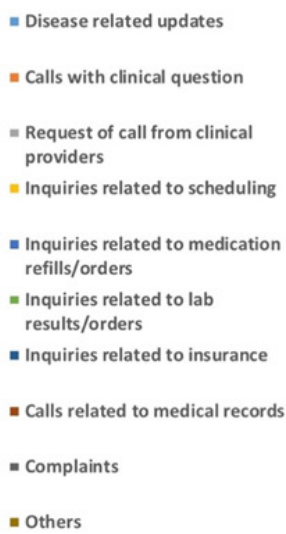

- others
May 2017

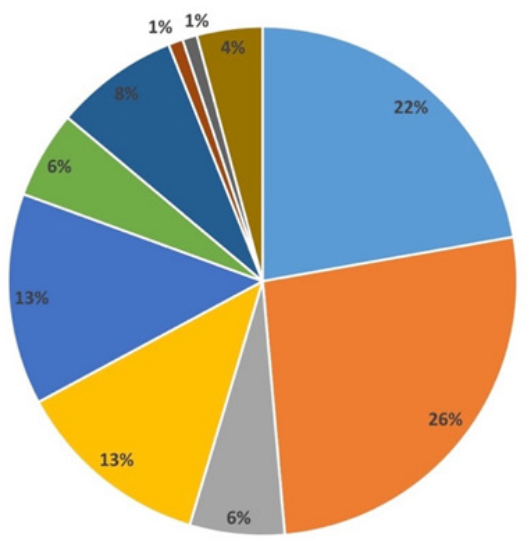

Figure 2 Percentage distribution of the calls related to each category in the respective months.

Our findings are similar to previous reports. ${ }^{7,8}$ We observed a significant decrease in the number of phone calls after implementation of our interventions.

Despite the recent explosion of health information through the Internet-based media, patients still prefer their physicians as their most desired source of information..$^{9,10}$ Oral information provided during the office visits can be inadequate, with studies showing that patients often recall less than $35 \%$ of information provided orally by health professionals. ${ }^{11}$ While patients would prefer having direct access to their physicians for information regarding their diseases, this raises several concerns, including putting additional demands on physicians' already busy schedule and lack of remuneration for these services. Several randomized studies have shown that well-structured and tailored written communication tools obtained from care provider are effective and increase patients' understanding of the disease. ${ }^{12-17}$
Written information for drugs used on a long- term basis may improve compliance in addition to patients' knowledge of less commonly known information, such as precautions, side effects, or special directions. Written materials also reinforce, and supplement oral education provided during clinical encounters. ${ }^{18}$ Given the chronic nature of IBD, patient education with help of interactive written brochures, not only about the medical disease but also about the logistics of medication administration and insurance issues appears to be a good investment and can significantly reduce the number of calls to the clinic.

Electronic patient portal linked to electronic health record has been introduced in the last two decades. Center for Medicare and Medicaid Services has listed this as stage 2 objective for meaningful use of electronic health records. ${ }^{19}$ This incentive has led many health systems to implement electronic patient portals in the recent years. 
Such portals give patients access to their electronic health record and the ability to communicate with their providers. They also allow the patients to view and modify list of medications and allergies, report nonadherence, side effects and other medication-related problems. The ease of online refills through portals has shown to improve compliance with patients with chronic medical problems. ${ }^{20}$ A survey has revealed that it helps patients understand their medical history better due to the availability of all the information in one place. (21) Some studies have also shown decrease in office visits and an increase in preventative medicine. ${ }^{22}$ Novel technologies like smart phone applications assisting patients with self-monitoring vitals and symptoms and communicating with healthcare provides have shown promising results in improving outcomes. ${ }^{23-26}$ However, only up to one-third of the patients use the electronic portal, ${ }^{21,27}$ and barriers to its use are not entirely understood yet. ${ }^{28}$ Communication of nonurgent issues with health providers by of electronic health portal helps decrease the time burden on the IBD call center which likely results in better triaging of the urgent medical issues over phone. The use of this potentially transformative technology needs to be strongly encouraged and promoted, especially in the management of chronic diseases such as IBD.

U.S. health care have high administrative costs, contributed significantly by physicians' offices interaction with health insurance companies. ${ }^{29}$ In the last few decades, physicians in the US and their office staff have experienced tremendous increase in time-consuming requirements of prior authorization of medications, medical tests and certain other clinical services. ${ }^{30-32}$ These requirements can be particularly cumbersome when dealing with drugs that are expensive or warrant close monitoring, newer therapies for chronic diseases or therapies for rare diseases; known as specialty pharmaceuticals. In response to specialized handling and administrative requirements with these therapies, a new pharmaceutical sector called specialty pharmacy has emerged. ${ }^{33-36}$ The specialty pharmacies provide services for specialty pharmaceuticals such as storage, handling and delivery, train and educated the patients and assist with administrative and reimbursement process. ${ }^{34,35}$ Given the chronic nature of the disease, complexity of management and frequent use of expensive 'biologics', ${ }^{37}$ managing insurance related administrative workload in a high volume IBD call center can be challenging. In our experience, partnering with specialty pharmacies and infusion centers helped share this burden and decrease the administrative demands on clinical providers immensely, leading to more efficient delivery of the IBD medications.

Another interesting finding in our study is that a significant number of calls (calls related to categories ${ }^{4,7-9}$ did not need direct input from clinical providers. These results are similar to previous studies. ${ }^{7,8} \mathrm{~A}$ recent practice management article has described midlevel providers such as nurse practitioners and physician assistants as an essential part of a dedicated IBD practice..$^{38}$ Addition of nurse practitioner to the team leads to improvement in overall care and has been shown to decrease health care costs and improve patient outcomes such as avoidance of ER visits/hospitalizations, decrease in outpatient visits and patient satisfaction. ${ }^{40-42}$ Nurse driven protocols targeting top clinical concerns for telephone calls can decrease physician burden and likely expedite care. Additionally, a significant portion of the calls could be addressed through triage of these phone calls to support personnel with tools and skills to obtain insurance authorizations, refill chronic medications, make changes in appointments and release or receive medical records, without needing assessment by the clinical providers. ${ }^{8,43}$

There are some limitations to our study, given the retrospective design. We identified female Crohn's patients as the largest subset of callers. Large prospective studies observing additional demographic information to clarify the "frequent caller" patient would help physicians target certain groups who appear to have more healthcare needs. Due to the retrospective nature of the study, we do not have the information about the actual time spent addressing each phone call. This would be useful to further elucidate the overall workflow burden of each type of inquiry, i.e., the proportion of all follow-up time taken by a subtype of inquiry. Lastly, assessment of any changes in the management outcomes would also help further characterize the clinical effect of our interventions.

\section{Conclusions}

Call center-based communication for chronic disease such as IBD requires significant investment of time and is associated with high administrative burden. An analysis of the calls to identify common reasons for patients' calls is an important step to address this issue. Focus on patient education with improvised methods, partnership with specialty pharmacies and outpatient infusion centers, encouragement of electronic communication via internet for non-urgent issues, nursedriven protocol for common concerns and appropriate triage of phone calls to administrative support personnel can help decrease this burden and improve the patient experience.

\section{Author contributions}

Anand Nath and Tenzin Choden participated in literature review, in collection of clinical and experimental data for this study, and in writing the manuscript. Aimee LeStrange participated in collection of clinical and experimental data and reviewed the manuscript. Mark Mattar conceived of and designed this study, and reviewed, edited, and finalized the manuscript. All authors read and approved the final paper.

\section{Source of funding}

No funding was received for this study.

\section{Informed consent statement}

Consent was not obtained but the presented data are anonymized, and risk of identification is low.

\section{Conflicts of interest}

All authors declare that there is no conflict of interest to report regarding the publication of this paper.

\section{References}

1. Molodecky NA, Soon IS, Rabi DM, et al. Increasing incidence and prevalence of the inflammatory bowel diseases with time, based on systematic review. Gastroenterology. 2012;142(1):46-54. e4

2. Mehta F. Report: economic implications of inflammatory bowel disease and its management. Am J Manag Care. 2016;22(3 Suppl):S51-S60.

3. Kappelman MD, Rifas-Shiman SL, Porter CQ, et al. Direct Health Care Costs of Crohn's Disease and Ulcerative Colitis in United States Children and Adults. Gastroenterology. 2008;135(6):1907-1913.

4. Verhoef MJ, Sutherland LR. Outpatient health care utilization of patients with inflammatory bowel disease. Dig Dis Sci.1990;35(10):1276-1280.

5. Castiglione F, Imperatore N, Testa A, et al. Efficacy of a "contact centerbased communication" in optimizing the care of inflammatory bowel diseases. Dig Liver Dis. 2016;48(8):869-873. 
6. Marklund B, Bengtsson C. Medical advice by telephone at Swedish health centres: who calls and what are the problems? Fam Pract. 1989;6(1):42-46.

7. Ramos-Rivers C, Regueiro M, Vargas EJ, et al. Association Between Telephone Activity and Features of Patients with in ammatory Bowel Disease. Clin Gastroenterol Hepatol. 2014;12(6): 986-994.e1.

8. Corral JE, Yarur AJ, Diaz L, et al. Cross-sectional analysis of patient phone calls to an inflammatory bowel disease clinic. Ann Gastroenterol. $2015 ; 28(3): 357$

9. Halpert A, Dalton CB, Palsson O, et al. Patient educational media preferences for information about irritable bowel syndrome (IBS). Dig Dis Sci. 2008;53(12):3184-3190.

10. Drossman DA, Morris CB, Schneck S, et al. International survey of patients with IBS: symptom features and their severity, health status, treatments, and risk taking to achieve clinical benefit. $J$ Clin Gastroenterol. 2009;43(6):541-550.

11. Ley, P. Satisfaction, compliance and communication. Br J Clin Psychol 1982;21(4):241-254.

12. Meade CD, McKinney WP, Barnas GP. Educating patients with limited literacy skills: the effectiveness of printed and videotaped materials about colon cancer. Am J Public Health. 1994 Jan;84(1):119-121.

13. Trevena LJ, Davey HM, Barratt A, et al. A systematic review on communicating with patients about evidence. J Eval Clin Pract. 2006;12(1):13-23.

14. Funnell MM, Donnelly MB, Anderson RM, et al. Perceived effectiveness, cost, and availability of patient education methods and materials. The Diabetes Educator. 1992;18(2):139-145.

15. Griffin J, McKenna K, Tooth L. Discrepancy between older clients' ability to read and comprehend and the reading level of written educational materials used by occupational therapists. Am J Occup Ther. 2006;60(1):70-80.

16. Baker D, Roberts DE, Newcombe RG, Fox KA. Evaluation of drug information for cardiology patients. British Journal of Clinical Pharmacology. 1991;31, 525-531.

17. Walker A. Teaching the illiterate patient. Journal of Enterostomal Therapy. 1987;14, 83-86. 'seppi

18. Morris LA, Halperin JA. Effects of written drug information on patient knowledge and compliance: a literature review. Am J Public Health. 1979;69(1):47-52.

19. Promoting Interoperability. 2018.

20. Sarkar U, Lyles CR, Parker MM, et al. Use of the refill function through an online patient portal is associated with improved adherence to statins in an integrated health system. Med Care. 2014;52(3):194.

21. Turvey C, Klein D, Fix G, et al. Blue Button use by patients to access and share health record information using the Department of Veterans Affairs' online patient portal. J Am Med Inform Assoc. 2014;21(4):657663.

22. Kruse CS, Bolton K, Freriks G. The effect of patient portals on quality outcomes and its implications to meaningful use: a systematic review. $J$ Med Internet Res. 2015;17(2).

23. Bengtsson U, Kasperowski D, Ring L, et al. Developing an interactive mobile phone self-report system for self-management of hypertension. Part 1: Patient and professional perspectives. Blood Press. 2014;23(5):288-295.

24. Licskai C, Sands TW, Ferrone M. Development and pilot testing of a mobile health solution for asthma self-management: asthma action plan smartphone application pilot study. Can Respir J. 2013;20(4):301-306.
25. Atreja A, Rizk M. Capturing patient reported outcomes and quality of life in routine clinical practice: ready for prime time? Minerva Gastroenterol Dietol. 2012;58(1):19-24.

26. Brennan PF. Personal health technology can improve outcomes. Mod Healthc. 2014;44(8):27.

27. Wallace LS, Angier H, Huguet N, et al. Patterns of electronic portal use among vulnerable patients in a nationwide practice-based research network: from the OCHIN practice-based research network (PBRN). $J$ Am Board Fam Med. 2016;29(5):592-603.

28. Ancker JS, Barrón Y, Rockoff ML, et al. Use of an electronic patient portal among disadvantaged populations. J Gen Intern Med. 2011;26(10):1117-1123.

29. Casalino LP, Nicholson S, Gans DN, et al. What does it cost physician practices to interact with health insurance plans? Health Aff. 2009;28(4):W533-W543.

30. Morley CP, Badolato DJ, Hickner J, et al. The impact of prior authorization requirements on primary care physicians' offices: report of two parallel network studies. J Am Board Fam Med. 2013;26(1):93-95.

31. Wickizer TM, Lessler D. Utilization management: issues, effects, and future prospects. Annu Rev Public Health. 2002;23(1):233-254.

32. Raper JL, Willig JH, Lin HY, et al. Uncompensated medical provider costs associated with prior authorization for prescription medications in an HIV clinic. Clin Infect Dis. 2010;51(6):718-724.

33. Baldini CG, Culley EJ. Estimated cost savings associated with the transfer of office-administered specialty pharmaceuticals to a specialty pharmacy provider in a medical injectable drug program. J Manag Care Pharm. 2011;17(1):51-59.

34. Edelman B. Specialty pharmacy booms, but for how long? Biotechnol Healthc. 2004;1(1):31-37.

35. Hesselgrave BL. Helping to manage the high cost of rare diseases. Manag Care Q. 2003;11(1):1-6.

36. Schwartz RN, Eng KJ, Frieze DA, et al. NCCN Task Force report: specialty pharmacy. J Natl Compr Canc Netw. 2010;8Suppl 4:S1-12.

37. Gleason PP, Alexander GC, Starner CI, et al. Health plan utilization and costs of specialty drugs within 4 chronic conditions. J Manag Care Pharm. 2013;19(7):542-548.

38. Kane S. Establishing an inflammatory bowel disease practice in an accountable world. Clin Gastroenterol Hepatol. 2012;10(12):13011304.

39. Belling R, McLaren S, Woods L. Specialist nursing interventions for inflammatory bowel disease. Cochrane Database Syst Rev. 2009;4:CD006597.

40. Leach P, De Silva M, Mountifield R, et al. The effect of an inflammatory bowel disease nurse position on service delivery. J Crohns Colitis. 2013;8(5):370-374

41. Nightingale AJ, Middleton W, Middleton SJ, et al. Evaluation of the effectiveness of a specialist nurse in the management of inflammatory bowel disease (IBD). Eur J Gastroenterol Hepatol. 2000;12(9):967-973.

42. Kapur A, Divakaran S, Walsh C, et al. O-008 YI The Impact of A Nurse Navigator Program on Telephone Calls and Emergency Department Utilization Among IBD Patients. Inflamm Bowel Dis. 2017;23(Suppl 1):S3.

43. Christino MA, Matson AP, Fischer SA, et al. Paperwork versus patient care: a nationwide survey of residents' perceptions of clinical documentation requirements and patient care. J Grad Med Educ. 2013;5(4):600-604. 